Supplement of The Cryosphere, 13, 1729-1741, 2019

https://doi.org/10.5194/tc-13-1729-2019-supplement

(c) Author(s) 2019. This work is distributed under

the Creative Commons Attribution 4.0 License.

(c) (1)

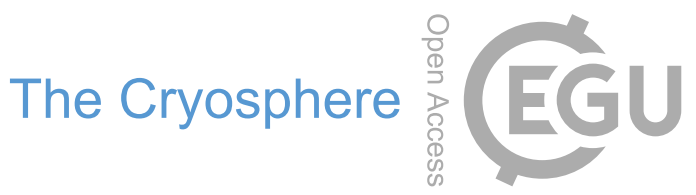

Supplement of

\title{
Automatically delineating the calving front of Jakobshavn Isbræ from multitemporal TerraSAR-X images: a deep learning approach
}

Enze Zhang et al.

Correspondence to: Enze Zhang (zhangenze@link.cuhk.edu.hk)

The copyright of individual parts of the supplement might differ from the CC BY 4.0 License. 


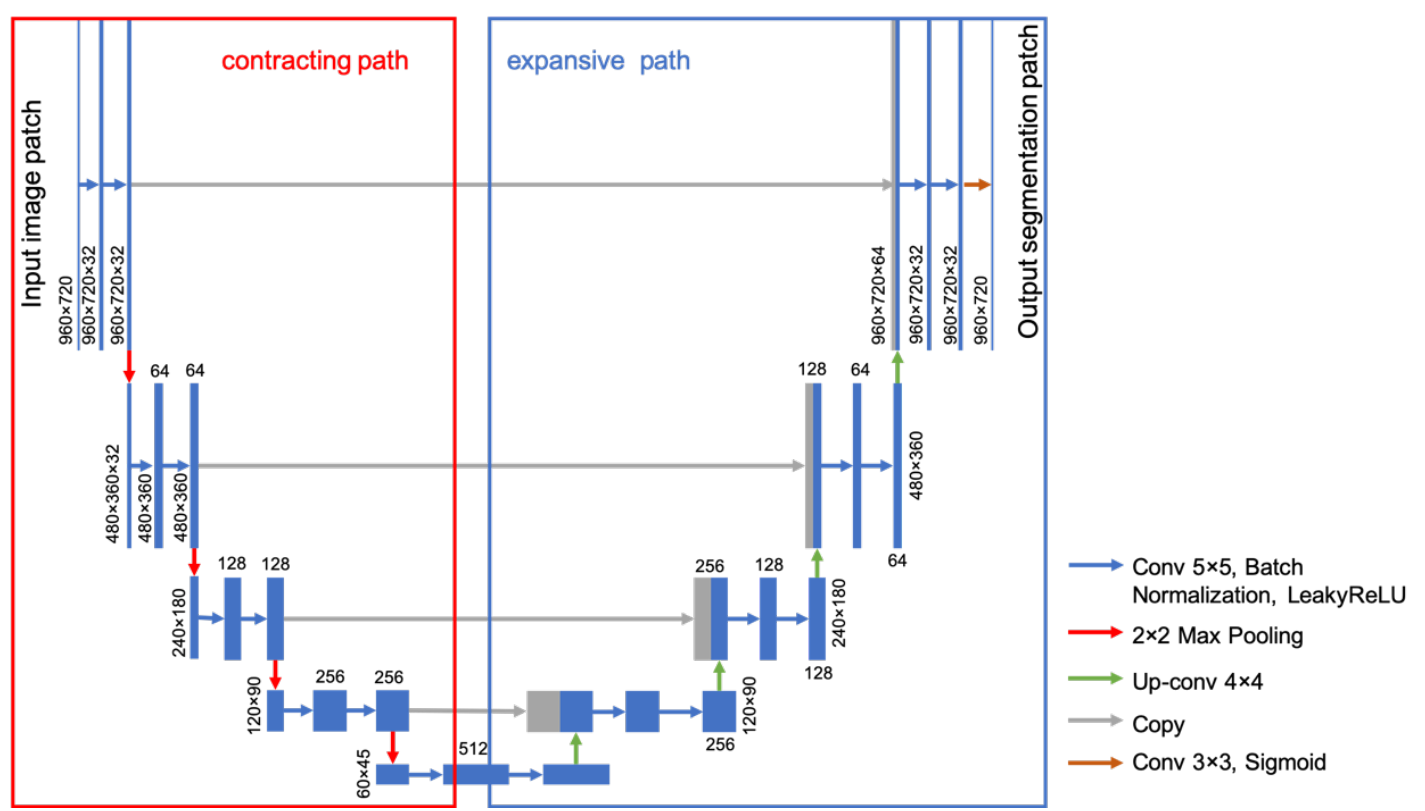

Figure S1. The Architecture of the U-Net. The red box indicates the contracting path and the blue box indicates the expansive path. Each solid blue box corresponds to a multi-channel feature map. Gray solid boxes represent copied feature maps. The length, width, and height of each layer correspond to the pixel dimensions and the number of feature channels respectively. Arrows with different colors denote the different operations.

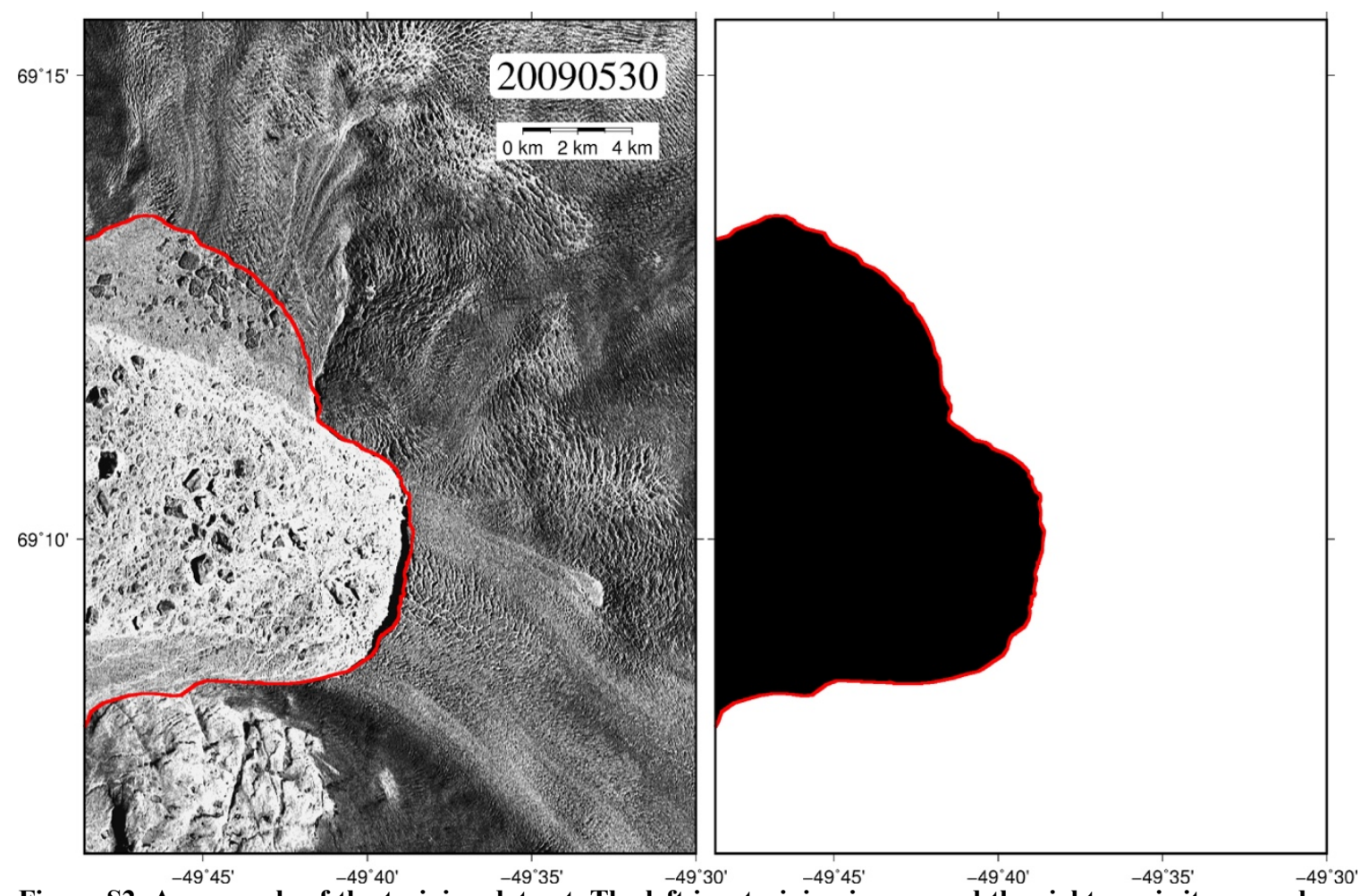

Figure S2. An example of the training dataset. The left is a training image, and the right one is its ground truth image where the black region is ice mélange and the white region is non-ice mélange (including both glacier and bedrock). The red line is the calving front we delineate manually. 

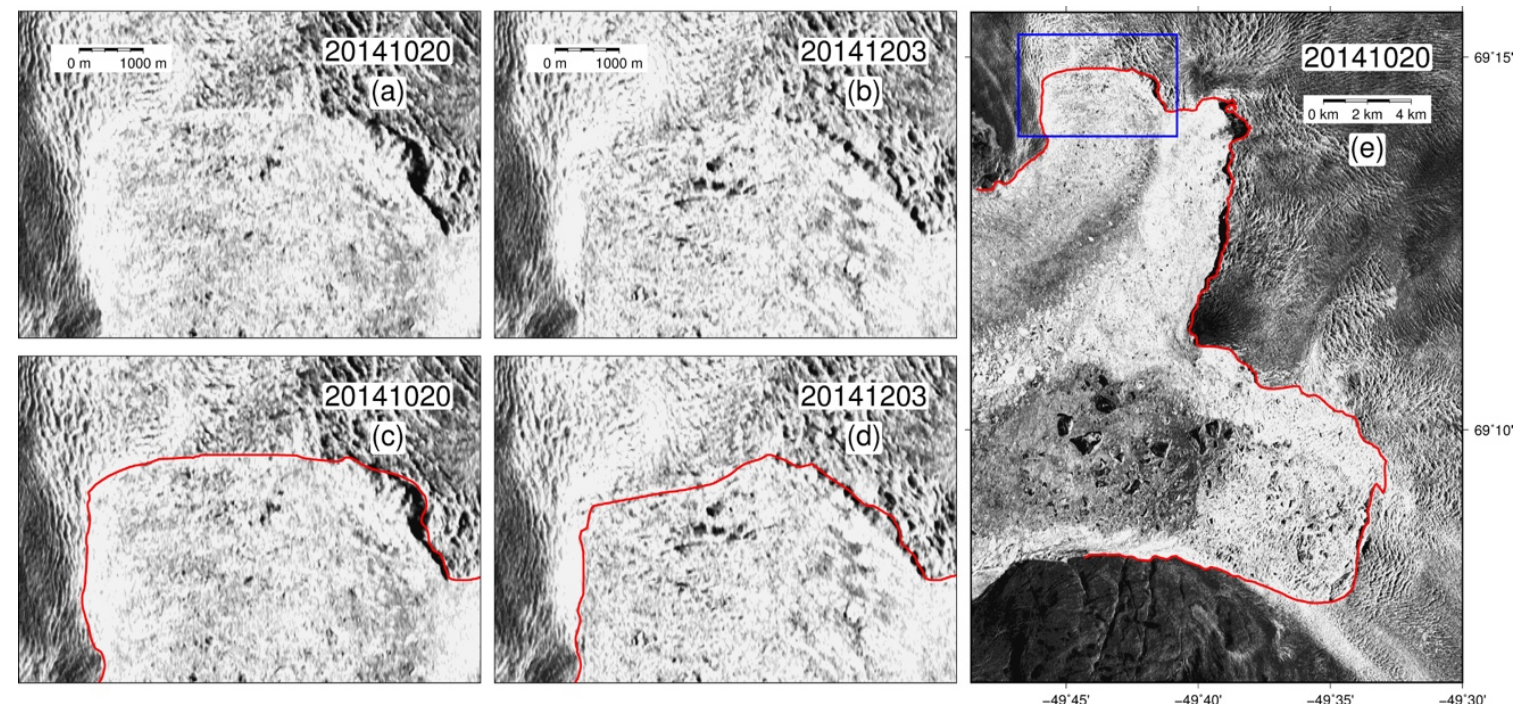

Figure S3. An example showing how we use a reference image to delineate a smooth calving front on a winter image with obscure boundaries. (a) is the reference image and (b) is the image with obscure boundaries. (c) and (d) show the manually delineated calving fronts. The blue box in (e) shows the location of (a)-(d).

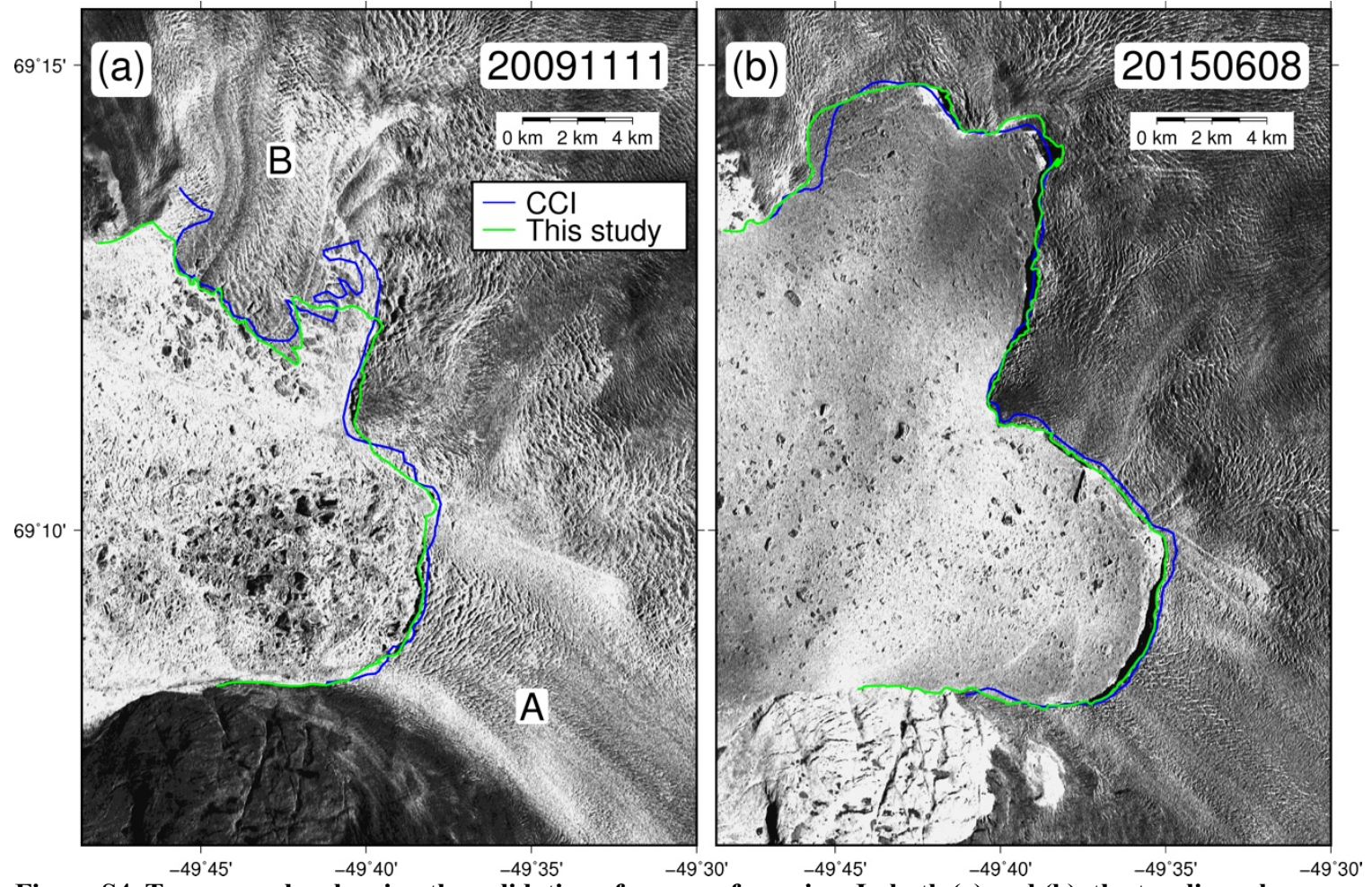

Figure S4. Two examples showing the validation of re-georeferencing. In both (a) and (b), the two lines show the manually delineated calving fronts from ours and the CCI. 


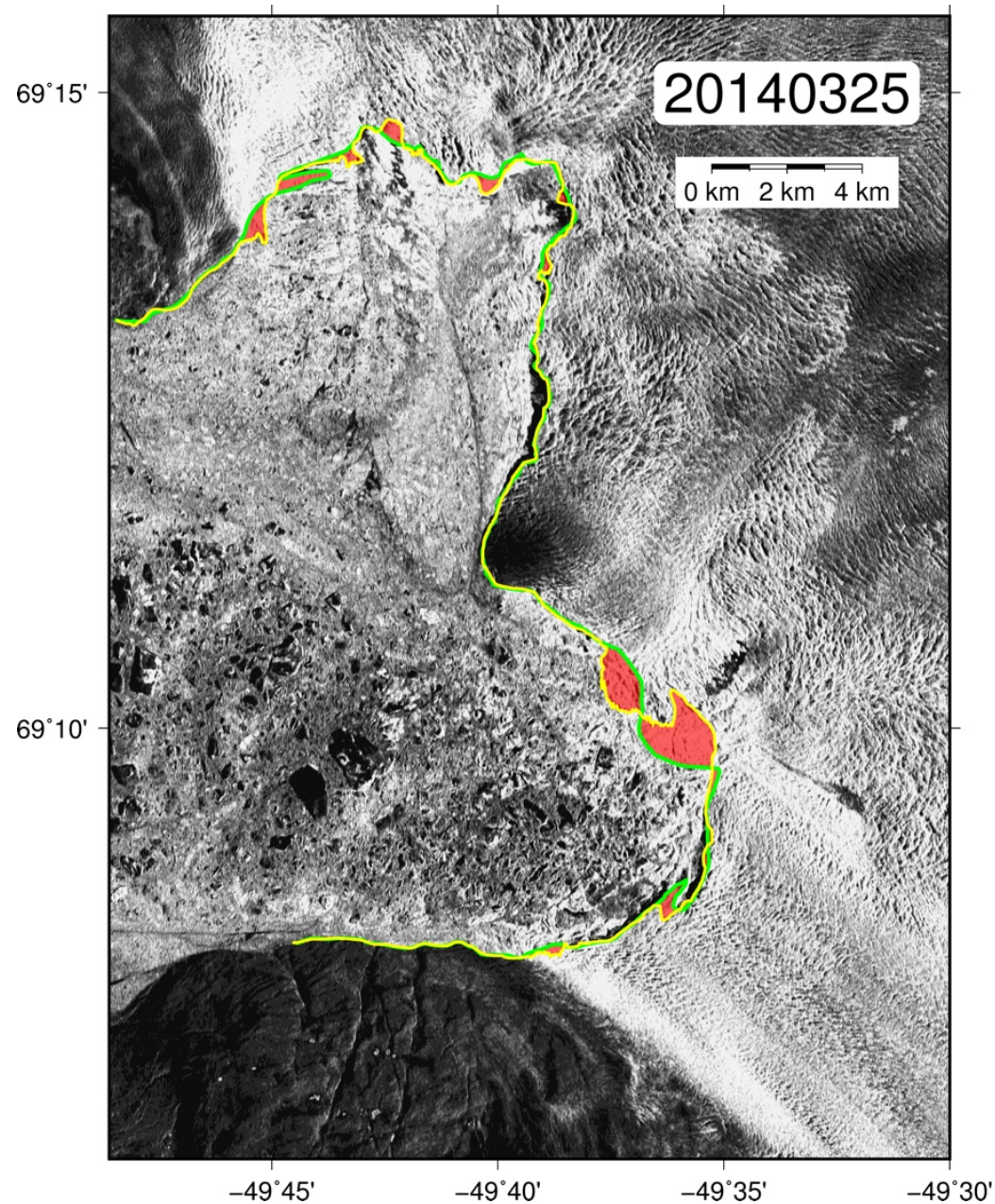

Figure S5. An example of error estimation of the network-delineation. The green line is the calving front we manually delineate. The yellow line is the calving front delineated by the deep-learning network. The red zone indicates the error of our results, which is the area circled by the green and yellow lines.

Movie S1. A movie showing the calving front positions from April $16^{\text {th }}, 2009$ to December $2^{\text {rd }}, 2015$. The red curve indicates the calving fronts delineated by our deep learning network.

DOI: https://doi.pangaea.de/10.1594/PANGAEA.897062

Movie S2. A movie showing the calving front variation of Branch A and the cross-section between the calving front and the profile. Two green lines indicate the bed elevation profiles. The red line shows the calving front position.

DOI: https://doi.pangaea.de/10.1594/PANGAEA.897080

Movie S3. Similar to Movie S2 but for Branch B.

DOI: https://doi.pangaea.de/10.1594/PANGAEA.897063 
Table S1. List of the TerraSAR-X images used in this study. ' $D$ ' and ' $A$ ' refer to the descending and ascending orbits, respectively, in the Orbit direction column. The orbit numbers represent the unique ID for each image. We also indicate whether each image is used as a test ' 0 ' or train-validation data ' 1 ' in the column 'Test/Train'.

\begin{tabular}{|c|c|c|c|c|c|c|c|}
\hline Date & $\begin{array}{c}\text { Orbit } \\
\text { direction }\end{array}$ & $\begin{array}{c}\text { Orbit } \\
\text { number }\end{array}$ & Test/Train & Date & $\begin{array}{c}\text { Orbit } \\
\text { direction }\end{array}$ & $\begin{array}{c}\text { Orbit } \\
\text { number }\end{array}$ & Test/Train \\
\hline 20090416 & $\mathrm{D}$ & 10192 & 1 & 20120911 & $\mathrm{~A}$ & 29085 & 0 \\
\hline 20090427 & $\mathrm{D}$ & 10359 & 1 & 20120921 & $\mathrm{D}$ & 29230 & 0 \\
\hline 20090508 & D & 10526 & 1 & 20121003 & A & 29419 & 1 \\
\hline 20090519 & $\mathrm{D}$ & 10693 & 1 & 20121014 & A & 12856 & 0 \\
\hline 20090530 & D & 10860 & 1 & 20121104 & D & 29898 & 1 \\
\hline 20090610 & $\mathrm{D}$ & 11027 & 1 & 20121115 & $\mathrm{D}$ & 30065 & 0 \\
\hline 20090621 & $\mathrm{D}$ & 11194 & 1 & 20121207 & $\mathrm{D}$ & 30399 & 1 \\
\hline 20090702 & $\mathrm{D}$ & 11361 & 0 & 20121219 & $\mathrm{~A}$ & 30588 & 0 \\
\hline 20090713 & $\mathrm{D}$ & 11528 & 1 & 20130109 & D & 30900 & 1 \\
\hline 20090724 & $\mathrm{D}$ & 11695 & 1 & 20130120 & $\mathrm{D}$ & 31067 & 0 \\
\hline 20090804 & $\mathrm{D}$ & 11862 & 0 & 20130211 & $\mathrm{D}$ & 31401 & 1 \\
\hline 20090815 & $\mathrm{D}$ & 12029 & 1 & 20130222 & $\mathrm{D}$ & 31568 & 0 \\
\hline 20090826 & $\mathrm{D}$ & 12196 & 0 & 20130306 & $\mathrm{~A}$ & 31757 & 0 \\
\hline 20090906 & $\mathrm{D}$ & 12363 & 0 & 20130317 & $\mathrm{~A}$ & 31924 & 1 \\
\hline 20090917 & $\mathrm{D}$ & 12530 & 0 & 20130407 & $\mathrm{D}$ & 32236 & 0 \\
\hline 20090928 & $\mathrm{D}$ & 12697 & 0 & 20130418 & $\mathrm{D}$ & 32403 & 0 \\
\hline 20091009 & $\mathrm{D}$ & 12864 & 0 & 20130510 & $\mathrm{D}$ & 32737 & 0 \\
\hline 20091020 & $\mathrm{D}$ & 13031 & 0 & 20130522 & A & 32926 & 1 \\
\hline 20091031 & $\mathrm{D}$ & 13198 & 1 & 20130613 & $\mathrm{~A}$ & 33260 & 1 \\
\hline 20091111 & $\mathrm{D}$ & 13365 & 0 & 20130624 & A & 33427 & 0 \\
\hline 20091122 & $\mathrm{D}$ & 13532 & 1 & 20130705 & $\mathrm{~A}$ & 33594 & 1 \\
\hline 20091203 & D & 13699 & 0 & 20130727 & A & 33928 & 0 \\
\hline 20091214 & $\mathrm{D}$ & 13866 & 1 & 20130817 & $\mathrm{D}$ & 34240 & 1 \\
\hline 20091225 & D & 14033 & 1 & 20130828 & D & 34407 & 0 \\
\hline 20100105 & $\mathrm{D}$ & 14200 & 0 & 20130908 & $\mathrm{D}$ & 17844 & 0 \\
\hline 20100116 & $\mathrm{D}$ & 14367 & 1 & 20130920 & $\mathrm{~A}$ & 18033 & 0 \\
\hline 20100207 & $\mathrm{D}$ & 14701 & 0 & 20131012 & $\mathrm{~A}$ & 18367 & 0 \\
\hline 20100218 & $\mathrm{D}$ & 14868 & 1 & 20131023 & $\mathrm{~A}$ & 18534 & 1 \\
\hline 20100312 & $\mathrm{D}$ & 15202 & 1 & 20131114 & $\mathrm{~A}$ & 18868 & 1 \\
\hline 20100323 & $\mathrm{D}$ & 15369 & 0 & 20131125 & $\mathrm{~A}$ & 19035 & 0 \\
\hline 20100414 & $\mathrm{D}$ & 15703 & 1 & 20131206 & $\mathrm{~A}$ & 19202 & 1 \\
\hline 20100425 & $\mathrm{D}$ & 15870 & 0 & 20131217 & $\mathrm{~A}$ & 19369 & 0 \\
\hline 20100506 & $\mathrm{D}$ & 16037 & 0 & 20140129 & $\mathrm{D}$ & 36745 & 1 \\
\hline 20100517 & $\mathrm{D}$ & 16204 & 1 & 20140303 & $\mathrm{D}$ & 37246 & 0 \\
\hline 20100619 & D & 16705 & 1 & 20140314 & D & 37413 & 1 \\
\hline 20100630 & $\mathrm{D}$ & 16872 & 0 & 20140325 & $\mathrm{D}$ & 37580 & 0 \\
\hline 20100711 & $\mathrm{D}$ & 17039 & 1 & 20140405 & $\mathrm{D}$ & 21017 & 0 \\
\hline 20100722 & $\mathrm{D}$ & 17206 & 1 & 20140416 & $\mathrm{D}$ & 21184 & 1 \\
\hline 20100904 & $\mathrm{D}$ & 17874 & 1 & 20140427 & $\mathrm{D}$ & 38081 & 0 \\
\hline 20100915 & $\mathrm{D}$ & 18041 & 0 & 20140610 & $\mathrm{D}$ & 38749 & 1 \\
\hline 20101007 & $\mathrm{D}$ & 1645 & 1 & 20140621 & $\mathrm{D}$ & 38916 & 0 \\
\hline 20101018 & $\mathrm{D}$ & 18542 & 0 & 20140702 & $\mathrm{D}$ & 39083 & 0 \\
\hline 20101109 & $\mathrm{D}$ & 18876 & 1 & 20140703 & $\mathrm{~A}$ & 39105 & 1 \\
\hline 20101120 & $\mathrm{D}$ & 19043 & 0 & 20140714 & $\mathrm{~A}$ & 39272 & 1 \\
\hline 20101201 & $\mathrm{D}$ & 19210 & 1 & 20140725 & $\mathrm{~A}$ & 39439 & 1 \\
\hline 20101223 & $\mathrm{D}$ & 19544 & 0 & 20140804 & $\mathrm{D}$ & 39584 & 1 \\
\hline 20110103 & $\mathrm{D}$ & 19711 & 1 & 20140918 & $\mathrm{~A}$ & 40274 & 0 \\
\hline 20110125 & $\mathrm{D}$ & 20045 & 0 & 20140928 & $\mathrm{D}$ & 40419 & 1 \\
\hline 20110216 & $\mathrm{D}$ & 20379 & 0 & 20141009 & $\mathrm{D}$ & 23856 & 0 \\
\hline 20110310 & D & 20713 & 0 & 20141020 & $\mathrm{D}$ & 24023 & 1 \\
\hline 20110321 & $\mathrm{D}$ & 20880 & 0 & 20141111 & $\mathrm{D}$ & 41087 & 1 \\
\hline 20110412 & $\mathrm{D}$ & 21214 & 1 & 20141203 & $\mathrm{D}$ & 41421 & 0 \\
\hline 20110423 & $\mathrm{D}$ & 21381 & 0 & 20141214 & $\mathrm{D}$ & 41588 & 1 \\
\hline 20110515 & $\mathrm{D}$ & 21715 & 1 & 20141225 & D & 41755 & 0 \\
\hline 20110526 & $\mathrm{D}$ & 21882 & 0 & 20150105 & $\mathrm{D}$ & 25192 & 0 \\
\hline 20110617 & $\mathrm{D}$ & 22216 & 0 & 20150116 & $\mathrm{D}$ & 25359 & 0 \\
\hline 20110720 & $\mathrm{D}$ & 22717 & 1 & 20150127 & $\mathrm{D}$ & 42256 & 1 \\
\hline 20110823 & $\mathrm{~A}$ & 6510 & 1 & 20150207 & $\mathrm{D}$ & 42423 & 0 \\
\hline
\end{tabular}




\begin{tabular}{|l|l|l|l||l|l|l|l|}
\hline 20110903 & A & 23407 & 1 & 20150219 & A & 42612 & 1 \\
\hline 20110914 & A & 23574 & 1 & 20150312 & D & 26194 & 1 \\
\hline 20111005 & D & 23886 & 0 & 20150323 & D & 43091 & 0 \\
\hline 20111016 & D & 24053 & 0 & 20150414 & D & 43425 & 1 \\
\hline 20111108 & A & 24409 & 0 & 20150425 & D & 43592 & 0 \\
\hline 20111118 & D & 24554 & 1 & 20150506 & D & 43759 & 0 \\
\hline 20111210 & D & 24888 & 0 & 20150517 & D & 43926 & 1 \\
\hline 20111221 & D & 25055 & 0 & 20150608 & D & 44260 & 0 \\
\hline 20120113 & A & 24409 & 1 & 20150619 & D & 44427 & 0 \\
\hline 20120123 & D & 25556 & 1 & 20150711 & D & 44761 & 0 \\
\hline 20120214 & D & 25890 & 0 & 20150722 & D & 44928 & 1 \\
\hline 20120225 & D & 26057 & 0 & 20150802 & D & 45095 & 0 \\
\hline 20120307 & D & 26224 & 0 & 20150813 & D & 45262 & 0 \\
\hline 20120329 & D & 26558 & 1 & 20150904 & D & 45596 & 0 \\
\hline 20120420 & D & 26892 & 1 & 20150926 & D & 45930 & 1 \\
\hline 20120524 & 12 & 27415 & 1 & 20151018 & D & 46264 & 0 \\
\hline 20120603 & D & 27560 & 0 & 20151029 & D & 46431 & 0 \\
\hline 20120614 & D & 27727 & 0 & 20151109 & D & 46598 & 1 \\
\hline 20120707 & 12 & 28083 & 1 & 20151120 & D & 46765 & 0 \\
\hline 20120718 & 12 & 11520 & 1 & 20151212 & D & 47099 & 1 \\
\hline 20120809 & 12 & 11854 & 1 & 20151223 & D & 47266 & 0 \\
\hline 20120820 & 12 & 28751 & 0 & & & & \\
\hline
\end{tabular}

Table S2. List of the geocoding difference between our results and the CCI products in terms of equivalent lengths, and the manual delineation error measured by comparing two sets of independent delineation results.

\begin{tabular}{|l|l|l|l|l|l|l|l|}
\hline Date (yyyymmdd) & 20091111 & 20100506 & 20110617 & 20140703 & 20140928 & 20150608 & Mean \\
\hline $\begin{array}{l}\text { Geocoding } \\
\text { Difference }(\mathrm{m})\end{array}$ & 167 & 95 & 77 & 84 & 115 & 87 & $104(\sim 17.3$ pixels $)$ \\
\hline $\begin{array}{l}\text { Manual Delineation } \\
\text { Error(m) }\end{array}$ & 40 & 71 & 12 & 22 & 34 & 18 & $33(\sim 5.5$ pixels $)$ \\
\hline
\end{tabular}

Table S3. Test error of each image and their means.

\begin{tabular}{|c|c||c|c||c|c|}
\hline Date & Error $(\mathrm{m})$ & Date & Error $(\mathrm{m})$ & Date & Error $(\mathrm{m})$ \\
\hline 20090702 & 26 & 20111221 & 48 & 20140427 & 72 \\
\hline 20090804 & 20 & 20120214 & 57 & 20140621 & 19 \\
\hline 20090906 & 20 & 20120225 & 27 & 20140702 & 38 \\
\hline 20090928 & 50 & 20120307 & 23 & 20140714 & 29 \\
\hline 20091009 & 66 & 20120614 & 20 & 20140725 & 14 \\
\hline 20091020 & 52 & 20120707 & 20 & 20140918 & 32 \\
\hline 20091111 & 34 & 20120820 & 15 & 20141009 & 27 \\
\hline 20091203 & 25 & 20120911 & 24 & 20141203 & 24 \\
\hline 20100105 & 38 & 20120921 & 38 & 20141225 & 31 \\
\hline 20100207 & 58 & 20121014 & 52 & 20150105 & 35 \\
\hline 20100323 & 65 & 20121115 & 34 & 20150116 & 31 \\
\hline 20100425 & 56 & 20121219 & 63 & 20150207 & 35 \\
\hline 20100506 & 57 & 20130120 & 59 & 20150323 & 42 \\
\hline 20100630 & 58 & 20130222 & 57 & 20150425 & 30 \\
\hline 20100915 & 12 & 20130306 & 58 & 20150506 & 48 \\
\hline 20101018 & 31 & 20130407 & 37 & 20150608 & 12 \\
\hline 20101120 & 42 & 20130418 & 32 & 20150619 & 22 \\
\hline 20101223 & 28 & 20130510 & 23 & 20150711 & 23 \\
\hline 20110125 & 21 & 20130624 & 39 & 20150802 & 20 \\
\hline 20110216 & 43 & 20130727 & 55 & 20150813 & 67 \\
\hline 20110310 & 22 & 20130828 & 32 & 20150904 & 45 \\
\hline 20110423 & 102 & 20130908 & 24 & 20151018 & 28 \\
\hline 20110526 & 61 & 20130920 & 34 & 20151029 & 48 \\
\hline 20110617 & 31 & 20131012 & 30 & 20151120 & 38 \\
\hline & & & & \\
\hline
\end{tabular}




\begin{tabular}{|l|l||l|l||c|c|}
\hline 20110903 & 19 & 20131125 & 19 & 20151223 & \multicolumn{2}{|c|}{32} \\
\hline 20111005 & 26 & 20131217 & 51 & & $38(6$ pixels $)$ \\
\hline 20111016 & 24 & 20140303 & 49 & Total Mean & $31(5$ pixels $)$ \\
\hline 20111108 & 67 & 20140325 & 74 & Summer Mean & $42(7$ pixels $)$ \\
\hline 20111210 & 35 & 20140405 & 38 & Winter mean & . \\
\hline
\end{tabular}

